\title{
Immunotherapy of brain cancers and neurological disorders
}

\begin{abstract}
Owing to the presence of the brain's protective barriers (BPBs) at the interface between the central nervous system (CNS) and the periphery, and their muted response to neuroinflammation, it has been widely assumed heretofore that the brain (and, more generally, the CNS) is immune-privileged. In other words, the brain's vaguely understood component of the immune system, as distinct from the rest of the body's immune system, is generally able to handle, treat, and overcome any adverse pathologies developing therein. However, in contrast to this earlier dogma, it is now evident that the CNS does contain immune capabilities, and that neuroinflammation is likely to play an important role in most, if not all, neurological disorders. In addition, the protective barriers of the brain contribute to the development of inflammation through either normal immune signaling, or disruption of the basic physiological barrier mechanisms.
\end{abstract}

It is difficult to distinguish between normal and disrupted barrier function because of the physiological changes that take place as part of normal development since childhood to aging and senescence. This is less difficult in a number of neurological disorders which have been clearly associated with the barriers' disruptions (opening, modification, distortion, etc.).

The BPBs consist of a number of structural and physiological elements (including especially tight junctions between adjacent barrier cells), and an array of influx and efflux transporters. However, despite these protective mechanisms, the capacity for immune-surveillance of the brain is maintained, and there is evidence of inflammatory signaling at the brain barriers that may be an important part of the body's response to damage or infection. This signaling system appears to change both with normal aging and during disease. Changes may affect organic phenomena (or diapedesis) of immune cells and active molecular transfer, or cause rearrangement of the tight junctions and an increase in passive permeability across barrier interfaces.

Here, in parallel with immunotherapy as an emergent therapy of cancer, I advance the opinion that brain immunotherapy should also become a similar therapy for brain cancers and neurological disorders. If proven, this approach would represent a paradigm shift in our therapeutic approach to brain cancer and neurological disorders.
Volume 8 Issue $6-2017$

Alain L Fymat
International Institute of Medicine and Science, USA

Correspondence: Alain L Fymat, International Institute of Medicine and Science, California, USA, Tel (760)

485-9149, Email alain.fymat@fiimas.org

Received: October II, 2017 | Published: November 29, 2017
Abbreviations: $\alpha$-syn, $\alpha$-synucleinopathies; $A \beta$, amyloid beta; AD, alzheimer's disease; ALL, acute lymphoblastic leukemia; ALS, amyotrophic lateral sclerosis; BBB, blood brain barrier; BPB, brain protective barriers; CAR, chimeric antigen receptor; CLL, chronic lymphocytic leukemia; CNS, central nervous system; CSF, cerebro-spinal fluid; CTLA, cytotoxic T-lymphocyte antigen; FDA, (U.S.) food and drug administration; MRI, magnetic resonance imaging; MS, multiple sclerosis; PD, parkinson's disease; NCI, (U.S.) national cancer institute; PBR, peripheral benzodiazepine receptor; $\mathrm{PD}$, programmed death; PET, positron emission tomography; RNA, ribonucleic acid; SCGS, single-cell genomic sequencing

\section{Diseases cited}

Acute Lymphoblastic Lymphoma; Alzheimer's Disease; Amyotrophic Lateral Sclerosis; Cancer (brain, colon, lung, melanoma, prostate, pancreatic, uterine); Dementia (with Lewy bodies; frontotemporal); Epilepsy; Huntington's disease; Melanoma; Meningitis; Meningoencephalitis; Multiple Sclerosis; Parkinson Disease

\section{Introduction}

In an earlier publication, ${ }^{1} \mathrm{I}$ emphasized that cancer is less an organ disease and more a disease of molecular mechanisms caused by mutations of specific genes. Beyond conventional treatment approaches to cancer (chemotherapy, surgery, radiation oncology, hormone therapy, gene therapy), I addressed more specifically immunotherapy. Simply stated, immunotherapy is the harnessing of the immune system to battle tumors. By targeting the immune system, not the tumor itself, immunotherapy represents an important paradigm shift in cancer treatment as it marks an entirely different way of treating cancer. However, immunotherapy has been successful in inducing long-term remissions of hard-to-treat cancers in only about one-third of patients. Despite the successes recounted in, ${ }^{1}$ it does not help everyone (for example, for patients with metastatic cancer, the odds remain long). We are largely clueless as to why more patients do not benefit and so researchers are racing to identify biomarkers that might offer answers, and to experiment with ways to make therapies more potent. Nonetheless, immunotherapy is an important emergent anti-cancer therapy. ${ }^{2-7}$

Immunotherapy has been applied with antibodies to cytotoxic T-lymphocyte antigen 4 (CTLA-4) (a new protein receptor on the surface of T-cells-a blocker). The application consisted in the novel twist on the technique, which consists in manipulating immunosuppression rather than strictly focusing on it (that is, 'blocking the blocker'). It has also been applied with programmed death (PD)-1 inhibitors (another blocker consisting of molecules 
expressed in dying T-cells, which also put a brake on T-cells). In the former case, acute lymphoblastic leukemia (ALL) and metastatic melanoma were treated (where it achieved complete and partial remissions, including, importantly, remission of small brain metastases like the ones previously identified in the case of former U.S. President Jimmy Carter). In the latter case, in cases of advanced unresectable melanoma, it was applied to lung, colon, prostate, uterine and pancreatic cancers.

In a companion publication, ${ }^{8}$ I considered personalized immunotherapeutic treatments involving genetically modified patient's T-cells to make them target tumor cells. The treatment, dubbed chimeric antigen receptor (CAR)-T therapy, is an important treatment avenue. Although still in clinical trials, CAR-therapy may target an assortment of cancers. It is therefore natural to surmise that brain immunotherapy, and eventually brain CAR-therapy, could also potentially achieve similar (even though likewise limited) successes $^{1,2,3,4,5,6,7,8,9}$

\section{Systemic immunotherapy}

In mounting an immune response to cancer, the body faces two major challenges: (a) It has difficulty distinguishing between normal and cancerous cells as the latter have sprung from the former; and (b) many cancer cells have developed various mechanisms to thwart the immune cells such as hiding from or/and even interfering with them.

As part of the innate mechanism of protecting healthy tissue, T-cells inspect cancer cells for the presence on their surface of two requisite molecules before they attack them: (a) MHC molecules (these are large protein complexes that cradle protein fragments or antigens), which are the targets presented to the T-cells by D-(dendritic) cells; and (b) a co-stimulatory ligand that triggers the signal for the T-cells to attack. In the absence of either (a) or (b), or both, the T-cells simply move on. Thus, cancer cells can fool T-cells in two ways corresponding respectively to (a) or/and (b) above, namely, stop producing MHC on their surfaces or display a form of co-stimulatory ligands that act as off-switches. To overcome these two eventualities, the CAR technology (see 8-12) has made it possible to genetically modify ("engineer") the T-cells in either of two ways so that, (a) instead of the D-(dendritic cells), the T-cells could home-in directly on antigens that may be abundant on cancer cells without necessarily being presented by the MHC molecules, or else (b) obviating altogether the need for the two-step process described earlier for attacking the cancer cells.

${ }^{1}$ Luthi-Carter R. Progress towards a vaccine for Huntington's disease. Mol Ther. 2003;7(5 Pt 1):569-570.

${ }^{2}$ Wolfgang WJ, Miller TW, Webster JM, et al. Suppression of Huntington's disease pathology in drosophila by human single-chain Fv antibodies. Proc Natl Acad Sci USA. 2005;102(32):11563-1568.

${ }^{3}$ Valerajan E, Masliah E. Immunotherapy for neurodegenerative diseases: Focus on $\alpha$-synucleinopathies. Pharmacol Ther. 2013;138(3):311-322.

${ }^{4}$ Asuni AA, Boutajangout A, Quartermain D, et al. Immunotherapy targeting pathological tau conformers in a tangle mouse model reduces brain pathology with associated functional improvements. J Neurosci. 2007;27(34):9115-9129. ${ }^{5}$ Blennow K, Zetterberg H, Rinne JO, et al. Effect of immunotherapy with Bapineuzumab on cerebrospinal fluid biomarker levels in patients with mild to moderate Alzheimer disease. Arch Neurol. 2012;69(8):1002-1010.

${ }^{6}$ Boutajangout A, Quartermain D, Sigurdsson EM. Immunotherapy targeting pathological tau prevents cognitive decline in a new tangle mouse model. J Neurosci. 2010;30(49):16559-16566.

${ }^{7}$ Gehrmann J, Matsumoto Y, Kreutzberg GW. Microglia: intrinsic immuneffector cell of the brain. Brain Res Rev. 1995;20(3):269-287.

${ }^{8}$ Schenk DB, Seubert P, Grundman M, et al. A beta immunotherapy: Lessons learned for potential treatment of Alzheimer's disease. Neurodegener Dis. 2005;2(5):255-260.

${ }^{9}$ Ubhi K, Masliah E. Recent advances in the development of immunotherapies for tauopathies. Exp Neurol. 2011;230(2):157-161.
Beginning with the earlier discoveries of CTL-4 and PD-1, immunotherapy has rapidly evolved during the past decade. With the accumulated knowledge of cancer biology and the immune response system, we have now progressed beyond the stage of merely working with what Nature offers us. We are no longer limited to the use of natural cancer biology but have progressed to the use of synthetic biology. We are able to overcome some natural limitations such as overcoming the need for MHC molecules or/and co-stimulatory ligands. Further, by genetically modifying the T-cells, we can direct them to home-in directly on antigens that may be abundant on cancer cells.

The technology has, however, its limitations as we currently know of no other molecule than CD19 that is a specific cancer target. Another limitation is its toxic effects, but we may be able in the future to attenuate if not eliminate their effects. The technology can also be refined so as not to exclusively depend on the presence of CD19, and can be tailored to patients so as to avoid the deleterious effects of cytokine overproduction (or "storms") that could be fatal for some. Lastly, the technology can be improved by combining it with other complementary therapeutic approaches in a multi-prong attack (such as a combination of some or all of the following: surgery, radiation therapy, chemotherapy, thermal ablation). The future is very promising, indeed, and we can foresee the CAR-T approach being successfully tried in many forms of cancer; this is actually beginning to take place! These several mechanisms have been described in detail in, ${ }^{8}$ including limitations, refinements, and improvements on the CAR-T technology.

\section{On the chimeric antigen receptor T-therapy}

With increased interest on genomic and personalized medicine, the question arose as to whether a personalized treatment involving genetically modified patient's T-cells could make them target tumor cells. The idea had its beginning in the pioneering work of Rosenberg (2010) at the U.S. National Cancer Institute, dubbed chimeric antigen receptor (CAR) T-therapy, which has now become an important treatment avenue. CAR-therapy is now the subject of numerous clinical trials in the hope that, like the antibodies, it can target an assortment of cancers.

The CAR- technology merges gene therapy, synthetic biology and cell biology in the laboratory. It involves the following four steps : (a) First, a batch of certain T-cells known to respond best to a given disease are extracted from the blood; (b) a custom-built virus is used to implant them with new genes; ${ }^{9}$ (c) cells are created that target a molecule (CD19) that is found on the surfaces of some cancers; and (d) the modified cells are then returned to the body, where their new DNA gives them a fresh set of targets to attack. It has been tested in dozens of studies $(\sim 1,000$ patients) in certain types of cancers (leukemia and lymphoma). Half or more of these patients are now living longer than expected and hundreds appear to be cancer-free. ${ }^{10}$

In step (b) above, several custom-built viruses could be theoretically employed. Thus, based on the well-known proclivity of HIV for infecting cells, Posey, June and Levine ${ }^{11}$ have used the HIV virus. The end result here is made up of two parts: (a) An antibody-like part of CAR that juts out of the cell surface to bind to the cancer antigen of choice; and (b) the rest of CAR plunges into and through the T-cell membrane to generate the proper signals, and activate the T-cells as soon as the cancer antigen is detected. They have further successfully employed it in a limited clinical trial against the surface protein CD19 (this protein is found on healthy B-cells as well as the malignant cells they might become). It was tested in cases of chronic lymphocytic 
leukemia (CLL) and an expanded repertoire of cancers. The results of the CAR T-therapy have been summarized in, ${ }^{8}$ particularly in the case of acute lymphoblastic lymphoma (ALL).

\section{A landmark moment for cancer immunotherapy with car T-therapy}

In its News Release of 30 August 2017, the U.S. Food \& Drug Administration (FDA) announced its approval of the CAR-T cell therapy to treat certain children and younger adults with B-cell acute lymphoblastic leukemia (ALL). This announcement has heralded the advent of the first gene therapy in the U.S. In a separate article, ${ }^{12}$ I discuss this historic action and try to elucidate its far reaching implications in the treatments of cancer and other serious and lifethreatening diseases.

CAR-therapy is now the subject of numerous clinical trials in the hope that, like the antibodies, it can target an assortment of cancers However, notwithstanding the successes achieved so far, CAR therapy may not benefit everyone. It needs to be extended to a larger population of patients, applied to treat a wide range of diseases including HIV, certain infections, immune deficiencies and autoimmune disorders, and cancers including solid tumors, and rendered more affordable.

\section{Brain immunotherapy}

The brain has its own specially tailored immune system separate from the rest of the body. Nonetheless, mobilizing cells from the systemic immune system does not always cause harm in the brain but, when well controlled, may in fact even help in coping with various brain pathologies. The peripheral immune response contributes to neuroinflammatory conditions as shown by Anthony et al., ${ }^{13}$ and indicated by Prof. Michal Schwartz from the Weizmann Institute of Science, Israel. ${ }^{14}$ This has been particularly well-established in multiple sclerosis (MS) and amyotrophic lateral sclerosis (ALS), stroke, and epilepsy among other disorders ${ }^{15-17}$ in which changes in the brain barriers' structure and function have been reported. These barriers play an important role in maintaining the homeostatic environment of the CNS, and damage to their various structural or/ and functional components may contribute significantly to disease etiology or progression. What is currently unclear is: (a) How the BPBs themselves contribute to inflammatory signaling in neurological disease? and (b) which specific barrier mechanisms are altered in response to inflammation?

Normal immune mechanisms in the CNS are often thought to be different from those of the periphery. For instance, the immune response in the brain can be substantial (e.g., in response to meningitis) but, by contrast, a loss of immunity is also reported (e.g., cerebral infections). It is the muted inflammatory response in the brain following injury that was the original rationale behind the concept of the CNS being an immune-privileged site.

\section{Neurodegenerative disorders}

With his research team, Schwartz ${ }^{15}$ further discovered a unique type of immune cell which, when orderly activated, could be the basis for the future treatment of Alzheimer's disease. This finding has led to the new concept wherein Alzheimer is viewed from a mechanistic understanding of the central role of microglial cells (these are the brain's unique immune cells). AD manifests itself by various neuronal pathological processes and a significant reduction in brain function. The beta-amyloid protein (or plaques) accumulates within and between brain cells. Due to these structural changes, chemical communication pathways (the synapses) are weakened and junctions between neuronal cells are $\operatorname{lost}^{10},{ }^{11}, 12,13,14,15,16,17,18,19,20,21,22,23,24,25,26,27,28,29,30,31,32,33,34$. In addition, the cytoskeletal proteins of the axons (the nerve fibers) lose their normal structure, impairing their function and causing massive death of nerve cells. The primary role of the microglia is to recognize, disassemble and dispose of various substances that do

${ }^{10}$ Citron M. Alzheimer's disease: Strategies for disease modification. Nat Rev Drug Discov. 2010;9(5):387-398.

${ }^{11}$ Corbett A, Smith J, Ballard C. New and emerging treatments for Alzheimer's disease. Expert Rev Neurother. 2012;12(5):535-543.

${ }^{12}$ Delrieu J, Ousset PJ, Caillaud C, et al. Clinical trials in Alzheimer's disease: Immunotherapy approaches. J Neurochem. 2012;120(Suppl 1):186-193.

${ }^{13}$ Farlow M, Arnold SE, van Dyck $\mathrm{CH}$, et al. Safety and biomarker effects of Solanezumab in patients with Alzheimer's disease. Alzheimers Dement. 2012;8:261-271.

${ }^{14}$ Fernández PL, Britton GB, Rao KS. Potential immunotargets for Alzheimer's disease treatment strategies. J Alzheimers Dis. 2012;33(2):297-312.

${ }^{15} \mathrm{Fu} \mathrm{HJ}$, Liu B, Frost JL, et al. Amyloid-beta immunotherapy for Alzheimer's disease. CNS Neurol Disord Drug Targets. 2010;9(2):197-206.

${ }^{16} \mathrm{Götz} \mathrm{J,} \mathrm{Ittner} \mathrm{A,} \mathrm{Ittner} \mathrm{LM.} \mathrm{Tau-targeted} \mathrm{treatment} \mathrm{strategies} \mathrm{in} \mathrm{Alzheimer's}$ disease. Br J Pharmacol. 2012;165(5):1246-1259.

${ }^{17}$ Hamilton RL. Lewy bodies in Alzheimer's disease: A neuropathological review of 145 cases using alpha-synuclein immunohistochemistry. Brain Pathol. 2000;10(3):378-384.

${ }^{18}$ Lasagna-Reeves CA, Castillo-Carranza DL, Jackson GR, et al. Tau oligomers as potential targets for immunotherapy for Alzheimer's disease and tauopathies. Curr Alzheimer Res. 2011;8(6):659-665.

${ }^{19}$ Lee VM, Otvos L, Schmidt ML, et al. Alzheimer's disease tangles share immunological similarities with multiphosphorylation repeats in the two large neurofilament proteins. Proc Natl Acad Sci USA. 1988;85:7384-7388.

${ }^{20}$ Lemere CA, Masliah E. Can Alzheimer's disease be prevented by amyloidbeta immunotherapy? Nat Rev Neurol. 2010;6(2):108-119.

${ }^{21}$ Mandelkow EM, Mandelkow E. Tau as a marker for Alzheimer's disease. Trends Biochem Sci. 1993;18(12):480-483.

${ }^{22}$ Menéndez-González M, Pérez-Piñera P, Martínez-Rivera $M$, et al. Immunotherapy for Alzheimer's disease: rational basis in ongoing clinical trials. Curr Pharm Des. 2011;17(5):508-520.

${ }^{23}$ Meraz-Ríos MA, Lira-De León KI, Campos-Peña V, et al. Tau oligomers and aggregation in Alzheimer's disease. J Neurochem. 2010;112:1353-1367.

${ }^{24}$ Morgan D. Immunotherapy for Alzheimer's disease. J Intern Med. 2011;269(1):54-63.

${ }^{25}$ Panza F, Frisardi V, Solfrizzi V, et al. Immunotherapy for Alzheimer's disease: from anti- $\beta$-amyloid to tau-based immunization strategies. Immunotherapy. 2012;4(2):213-238.

${ }^{26}$ Pul R, Dodel R, Stangel M. Antibody-based therapy in Alzheimer's disease. Expert Opin Biol Ther. 2011;11(3):343-357.

${ }^{27}$ Sigurdsson EM. Immunotherapy targeting pathological tau protein in Alzheimer's disease and related tauopathies. J Alzheimers Dis. 2008;15(2):157-168.

${ }^{28}$ Tarawneh R and Holtzman DM. Critical issues for successful immunotherapy in Alzheimer's disease: Development of biomarkers and methods for early detection and intervention. CNS Neurol Disord Drug Targets. 2009;8(2):144-159.

${ }^{29}$ Town Tn. Alternative Abeta immunotherapy approaches for Alzheimer's disease. CNS Neurol Disord Drug Targets. 2009;8(2):114-127.

${ }^{30}$ Wang YJ, Zhou HD, Zhou XF. Modified immunotherapies against Alzheimer's disease: toward safer and effective amyloid clearance. J Alzheimers Dis. 2010;21(4):1065-1075.

${ }^{31}$ Wilcock DM, Colton CA. Anti-amyloid-beta immunotherapy in Alzheimer's disease: Relevance of transgenic mouse studies to clinical trials. J Alzheimers Dis. 2000;15(4):555-569.

${ }^{32}$ Crunkhorn S. Neurodegenerative disease: Immunotherapy opportunity emerges for Alzheimer disease. Nat Rev Drug Discovery. 2016;15(3):158.

${ }^{33}$ Hoyos M. Neurodegenerative diseases: New kinase targets for Alzheimer's disease. Nat Rev Drug Discovery. 2013;12(10):739.

${ }^{34}$ Flight MH. Neurodegenerative disease: Tau immunotherapy targets transcellular propagation. Nat Rev Drug Discovery. 2013;12(12):904. 
not function properly in the brain, from dying cells to various cell debris and protein aggregates. Yet, microglial activity is under tight regulation to allow them to dispose of waste without harming adjacent healthy neurons that retain important information. From essential immune function to the risk of damaging healthy neurons due to hyperactivity, the microglia's activities are well balanced in young healthy individuals, but might become a disadvantage in aging adults and under certain neuropathological conditions.

A key question remains why the brain's own immune cells are not effective in repairing the damage associated with $\mathrm{AD}$ and other disease(s). Also, a significant obstacle to understanding the roles of immune cells in $\mathrm{AD}$ and other neurodegenerative diseases is the ability to accurately distinguish between similar cells with different functions and, thus, understand which is "friend" and which is "foe." For this purpose, Schwarz's team employed advanced single-cell genomic sequencing (SCGS) technology-a "genetic microscope" recently developed at the Weizmann's Institute - to fully sequence the genetic material of single immune cells, allowing them to identify their unique function, even when they are extremely rare. In their study, they sequenced the RNA content of all the immune cells in the brains of the Alzheimer's disease mouse model, repeated this experiment at different points in the disease progression, and compared the results with those from healthy mice. Clinical trials of this approach are urgently needed in light of the debilitating and even deadly effects of Alzheimer's and the promise of a solution by this approach.

Schwarz et al.'s discovery ${ }^{15}$ has now provided an answer to this question, along with a new research approach toward finding ways of treating the disease. These researchers studied a geneticallyengineered mouse model of $\mathrm{AD}$ whose genetic makeup includes five mutant human genes that cause an aggressive form of the disease. The brains of these mice exhibit features similar to those of humans suffering from dementia. These scientists' accomplishments are as explained above: (a) Discovery of a unique type of immune cell that could provide the basis of treatment for $\mathrm{AD}$; (b) answers the question of why the brain's own immune cells are not effective in repairing the damage associated with $\mathrm{AD}$ (and other) disease(s); (c) employed advanced single-cell genomic sequencing (SCGS) technology (a "genetic microscope") to fully sequence the genetic material of single immune cells, identifying their unique function, even when they are extremely rare; and (d) sequenced the RNA content of all the immune cells in the brains of the Alzheimer's disease mouse model, repeating this experiment at different points in the disease progression, and comparing the results with those from healthy mice.

\section{Immunotherapy for specific neurodegenerative disorders}

A number of laboratories are intensively exploring the use of immunotherapy in the much needed disease-modifying treatment of neurodegenerative disorders. ${ }^{18,19}$ While AD has been the focus of numerous immunotherapeutic studies, less attention has been paid to Parkinson's disease (PD) and other neurodegenerative disorders. The reason for this difference is that the amyloid beta $(A \beta)$ protein in $\mathrm{AD}$ is a secreted molecule that circulates in the blood and is readably recognized by antibodies. In contrast, $\alpha$-synucleinopathies $(\alpha$-syn), tau and other proteins involved in neurodegenerative diseases have been considered to be exclusively of intracellular nature. However, the recent discovery that toxic oligomeric versions of $\alpha$-syn and tau accumulate in the membrane and can be excreted to the extracellular environment has provided a rationale for the development of immunotherapeutic approaches for PD, dementia with Lewy bodies, fronto-temporal dementia, and other neurodegenerative disorders characterized by the abnormal accumulation of these proteins. Active immunization, passive immunization, and $\mathrm{T}$ cell-mediated cellular immunotherapeutic approaches have been developed targeting $\mathrm{A} \beta$, $\alpha$-syn and tau. Most advanced studies, including results from phase III clinical trials for passive immunization in $\mathrm{AD}$, have been recently reported. Results suggest that immunotherapy might be a promising therapeutic approach for neurodegenerative diseases that progress with the accumulation and propagation of toxic protein aggregates.

Immunizations that target specific types of immune responses are used commonly to prevent microbial infections. However, a range of immune responses may prove necessary to combat the ravages of neurodegenerative diseases. The goal is to eliminate the 'root' cause of neurodegenerative disorders, and misfolded aggregated proteins, while harnessing adaptive immune responses to promote neural repair. However, immunization strategies used to elicit humoral immune responses against aberrant brain proteins have yielded mixed success. While specific proteins can be cleared, the failures in halting disease progression revolve, in measure, around adaptive immune responses that promote autoreactive $\mathrm{T}$ cells and, as such, induce a meningoencephalitis, accelerating neurodegeneration. Thus, alternative approaches for protein clearance and neural repair are desired. How to harness the immune response in the setting of PD requires a thorough understanding of the role of immunity in human disease and the ways to modify such immune responses to elicit therapeutic gain $35,36,37,38,39,40,41,42,43,44,45,46,47,48,49,50$.

${ }^{35}$ Burton EJ, McKeith IG, Burn DJ, et al. Cerebral atrophy in Parkinson's disease with and without dementia: A comparison with Alzheimer's disease, dementia with Lewy bodies and controls. Brain. 2004;127(Pt 4):791-800.

${ }^{36}$ Lema Tomé CM, Tyson T, et al. Inflammation and $\alpha$-Synuclein's prionlike behavior in Parkinson's disease-Is there a link? Mol neurobiol. 2012;47(2):561-574.

${ }^{37}$ Lesage S, Brice A. Role of mendelian genes in "sporadic" Parkinson's disease. Parkinsonism Relat Disord. 2012;18(Suppl 1):S66-S70.

${ }^{38} \mathrm{Li}$ JY, Englund E, Holton JL, Soulet D, et al. Lewy bodies in grafted neurons in subjects with Parkinson's disease suggest host-to-graft disease propagation. Nat Med. 2008;14(5):501-503.

${ }^{39}$ Lücking CB, Brice A. Alpha-synuclein and Parkinson's disease. Cell Mol Life Sci. 2000;57(13-14):1894-1908.

${ }^{40}$ Luk KC, Kehm V, Carroll J, et al. Pathological $\alpha$-synuclein transmission initiates Parkinson-like neurodegeneration in nontransgenic mice. Science. 2012;338(6109):949-953.

${ }^{41} \mathrm{McCoy}$ MK, Cookson MR. Mitochondrial quality control and dynamics in Parkinson's disease. Antioxid Redox Signal. 2012;16(9):869-882.

${ }^{42}$ Rohn TT. Targeting alpha-synuclein for the treatment of Parkinson's disease. CNS Neurol Disord Drug Targets. 2012;11(2):174-179.

${ }^{43}$ Schneeberger A, Mandler M, Mattner F, et al. Vaccination for Parkinson's disease. Parkinsonism Relat Disord. 2012;18(Suppl 1):S11-S13.

${ }^{44}$ Schulz-Schaeffer WJ. The synaptic pathology of alpha-synuclein aggregation in dementia with Lewy bodies, Parkinson's disease and Parkinson's disease dementia. Acta Neuropathol. 2010;120(2):131-143.

${ }^{45}$ Spillantini MG, Goedert M. The alpha-synucleinopathies: Parkinson's disease, dementia with Lewy bodies, and multiple system atrophy. Ann N Y Acad Sci. 2000;920:16-27.

${ }^{46}$ Frost B and Diamond MI. Prion-like mechanisms in neurodegenerative diseases. Nat Rev Neurosci. 2010;11(3):155-159.

${ }^{47}$ Goedert M, Clavaguera F, Tolnay M. The propagation of prion-like protein inclusions in neurodegenerative diseases. Trends Neurosci. 2010;33(7):317-325.

${ }^{48}$ Lashuel HA, Hartley D, Petre BM, et al. Neurodegenerative disease: Amyloid pores from pathogenic mutations. Nature. 2002;418:291.

${ }^{49}$ Spillantini MG, Goedert M. Tau protein pathology in neurodegenerative diseases. Trends Neurosci. 1998;21(10):428-433.

${ }^{50}$ Ubhi K, Rockenstein E, Mante M, et al. Neurodegeneration in a transgenic mouse model of multiple system atrophy is associated with altered expression of oligodendroglial-derived neurotrophic factors. J Neurosci. 2010;30(18):6236-6246. 


\section{Case of metastatic melanoma}

Despite the best clinical efforts and breakthroughs in biotechnology, most patients diagnosed with advanced stage melanoma continue unfortunately to die from their disease. Reasons for this include: (a) Patients are often diagnosed at a time when their melanoma has already spread to other sites such as the chest cavity, bone, liver, and brain limiting the options for surgical excision and (b) the cancer cells are resistant or become resistant to chemotherapy drugs used to treat the patient. Resistance to one type of chemotherapy agent often rapidly leads to resistance against many other chemotherapy drugs. These reasons are the major causes of cancer progression that are usually discussed when considering treatment options for patients with disease that continues to grow and spread.

However, another important part of the body should be considered-the immune system. Scientists have clearly shown that melanoma cells produce a number of abnormal proteins or abnormal amounts of certain proteins found in normal melanoma cells. Normally, one would expect a patient to develop an immune response against these abnormal proteins found in their cancer and attack them much in the way we would fight off an infection from a foreign bacteria or virus. However, for reasons not fully understood, the immune system fails to respond adequately to these abnormal proteins and does not destroy the melanoma cells. Some scientists have proposed a new way to make the immune system recognize the cancer cells and encourage it to attack and destroy them. The approach consists in placing a mouse gene into human melanoma cancer cells so that those cells produce abnormal sugar patterns and stimulate the immune system to attack the melanoma. This strategy works well to kill other human cancer cells in the laboratory, but it needs to be tried in melanoma patients to see if it will be effective and to determine if such a treatment causes any side effects.

\section{Some clinical trials of interest}

Except for the first clinical trial, the other trials have been extracted from the National Cancer Institiute (NCI)'s website (www.nci.gov) in which all trial details can be found:

A. A phase 2 multiple ascending dose trial of Bapineuzumab in mild to moderate Alzheimer 's disease by Salloway S, Sperling R, Gilman S, Fox NC, Blennow K, Raskind M, et al. (2009). Neurology 73: 2061-2070.

B. An investigational immunotherapy study to evaluate safety and effectiveness in patients with melanoma that has spread to the brain treated with Nivolumab in combination with Ipilimumab followed by Nivolumab by itself". Patients with histologically confirmed malignant melanoma and asymptomatic brain metastases are eligible for the study.

C. Immunotherapy study for patients with stage IV melanoma.

Patients are given 4 injections of Ipilimumab, given 3 weeks apart $\mathrm{x} 4$ injections with or without HAM immunotherapy. This dual therapy is tested in patients with melanoma who have known stage $\mathrm{IV}$, metastatic melanoma. The underlying hypothesis is that this form of combinatorial immunotherapy will result in tumor stabilization or shrinkage, significant prolongation of progression-free, disease-free or overall survival compared to the use of Ipilimumab alone.

D. Carbon C 11 PBR-28 PET/MRI in Measuring Inflammation in Patients

This pilot clinical trial studies how well carbon C 11 periphera benzodiazepine receptor (PBR)-28 positron emission tomography $(\mathrm{PET}) /$ magnetic resonance imaging (MRI) works in measuring inflammation in patients with brain tumors undergoing treatment with chemoradiation or immunotherapy. Diagnostic procedures, such as carbon C 11 PBR-28 PET/MRI, done before, during, and after chemoradiation or immunotherapy may be able to detect changes in inflammation and help understand the role of inflammation in the treatment of cancer. The study's primary objectives are: (a) To determine whether PET imaging with [11C] PBR28 (carbon C 11 PBR-28) can measure inflammation related to immunotherapy in patients with brain tumors, specifically metastatic brain tumors and glioblastoma; and (b) to determine whether PET imaging with [11C] PBR28 can measure inflammation related to chemoradiation in patients with glioblastoma.

\section{Neuroinflammatory disorders}

In a typical adult inflammatory state, cells mediating the inflammatory response arrive at the site of inflammation or infection and release a large number of mediators that act to control the accumulation and activation of other cell types (both local and migrating). The key features of CNS inflammation include a range of responses including: (a) Glial activation, (b) edema, (c) major histocompatibility complex expression, (d) systemic acute phase response (general inflammation and acute phase protein synthesis), (e) complement activation, (f) synthesis of inflammatory mediators (e.g., cytokines, free radicals, prostaglandins), (g) expression of adhesion molecules, and (h) invasion of circulating immune cells. Due to the protective nature of both the $\mathrm{BBB}$ and the blood-CSF barriers, two questions remain: (1) Are mediators of the inflammatory response, captured within the CNS space, unable to be removed rapidly by the bloodstream? and (2) does the functional tightness of the barrier impede the entry of immune cells, thus slowing the immune response? Though the brain can mount its own defense by the activation of resident cells (such as astrocytes and microglia that are able to produce and secrete a number of cytokines), there is still major reliance on peripheral immune cells. There is a continuing argument that, given the limited regenerative capacity of the CNS, what is the balance between protection and damage to it that results from a neuroinflammatory response? The CNS is continuously monitored by resident microglia and blood-borne immune cells (such as macrophages, dendritic cells, and $\mathrm{T}$ cells) to detect damaging agents that would disrupt homeostasis and optimal functioning of neurons and glia.

\section{Interaction of the peripheral and the central nervous immune systems}

An important example of the interaction of peripheral immune cells with the CNS is the myeloid origin of the innate CNS immune cells-the microglia. Additionally, resident bone marrow-derived perivascular cells inhabit the perivascular space, which directly communicates with the CSF-filled subarachnoid space. These cells are able to respond rapidly to inflammatory injury and are continuously replaced by peripheral monocytes. It is therefore essential that the BPBs be able to facilitate entry of these cells into the CNS as part of normal function. However, this mechanism for facilitating peripheral immune cell entry to the CNS appears to be up-regulated in cases of neuroinflammation ${ }^{51}$ (e.g., during stroke and multiple sclerosis), and is associated with deleterious effects on pathology. There is, therefore, a fine line between a "helpful" inflammatory response to injury in the CNS and pathological neuroinflammatory state. Immune surveillance,

${ }^{51}$ Streit WJ, Mrak RE, Griffin WS. Microglia and neuroinflammation: A pathological perspective. J Neuroinflammation. 2004;1:14. 
in the absence of specific inflammatory signals is therefore likely to occur primarily through the blood-CSF barrier, facilitated by the specific composition of the junctions between epithelial cells. Importantly, junctional rearrangement appears to be an essential element of inflammation-induced cellular recruitment to the brain.

\section{Summary remarks}

A. The brain is surrounded by a complex array of barrier mechanisms comprised of (a) morphological (tight junctions), (b) biochemical, and (c) physiological (influx and efflux transporters) components that control and determine its internal environment. One important function is an interaction with the immune system. Any structural deficits within the barrier junctions that exist as a result of injury may increase the risk of early onset of neurodegenerative conditions.

B. Normal immune surveillance, which occurs primarily through the blood-CSF barrier, is facilitated by the specific composition of the junctions between epithelial cells. The rearrangement of the junctions is an essential element of inflammation-induced cellular recruitment to the brain.

C. Transporters present at brain barriers can be affected by inflammation, therefore contributing to changes in the CNS environment alone or in association with the changes produced by the activation of immune cells.

D. There is increasing evidence that the BPBs are able to mount a response to peripheral inflammation and that this vascular inflammatory response may itself contribute significantly to neuroinflammatory disease.

\section{Conclusion}

The brain barrier systems provide an essential interface between the periphery and the brain, and are involved in the communication of inflammatory signals between the two. Immunity is an active and fluid component of normal brain-barrier function. This function is likely altered under stress. More attention needs to be paid to changes in cellular-based barrier mechanisms, rather than focus on the integrity of tight junctions, which has been the emphasis of much of the research effort in this field so far. ${ }^{20,21}$

\section{Acknowledgments}

None.

\section{Conflicts of interest}

Author declares there are no conflicts of interest.

\section{References}

1. Fymat AL. Immunotherapy: An Emergent Anti-Cancer Strategy. $J$ Cancer Prev Curr Res. 2017;7(3):00233.

2. Kruse CA, Merchant RE. Cellular therapy of brain tumors: Clinical trials. Armonk, Futura Publ Co: USA; 1997. 487-504 p.
3. Paul DB, Kruse CA. Immunologic approaches to therapy for brain tumors. Curr Neurol Neurosci Rep. 2001;1(3):238-244.

4. Yang MY, Zetler PM, Prins RM, et al. Immunotherapy for patients with malignant glioma: From theoretical principles to clinical applications. Expert Rev Neurother. 2006;6(10):1481-1494.

5. Huse JT, Holland EC. Targeting brain cancer: Advances in the molecular pathology of malignant glioma and medulloblastoma. Nat Rev Cancer. 2010;10(5):319-331.

6. Xu X, Stockhammer F, Schmitt M. Cellular-based immunotherapies for patients with glioblastoma multiform". Clinical and Developmental Immunology. 2012;764213:15.

7. Reardon DA, Wucherpfennig KW, Freeman G, et al. An update on vaccine therapy and other immunotherapeutic approaches for glioblastoma. Expert Rev Vaccines. 2013;12(6):597-615.

8. Fymat AL. Synthetic Immunotherapy with Chimeric Antigen Receptors. J Cancer Prev Curr Res. 2017;7(5):00253.

9. Maude SL, Frey N, Shaw PA, et al. Chimeric Antigen Receptors T-Cells for Sustained Remissions in Leukemia. New Engl J Med. 2014;371(16):1507-1517.

10. Maus MV, Fraietta JA, Levine BL, et al. Adoptive Immunotherapy for Cancer or Viruses. Annual Review of Immunology. 2014;32:189-225.

11. Posey AD, June CH, Levine BL. Cancer Killers. Scientific American; 2017. 38-43 p.

12. Fymat AL. Cancer therapy with chimeric antigen receptors-A landmark moment for cancer immunotherapy. J Cancer Prev Curr Res; 2017.

13. Anthony DC, Couch Y, Losey $\mathrm{P}$, et al. The systemic response to brain Injury and disease. Brain Behav Immun. 2011;26(4):534-540.

14. Campbell SJ, Meier U, Mardiguian S, et al. Sickness behavior is induced by a peripheral CXC-Chemokine also expressed in multiple sclerosis and EAE. Brain Behav Immun. 2010;24(5):738-746.

15. Schwartz M. Weizmann Institute of Science: Israel; 2017.

16. Serres S, Anthony DC, Jiang Y, et al. Systemic inflammatory response reactivates immune-mediated lesions in rat Brain. $J$ Neurosci. 2009;29(15):4820-4828.

17. Auvin, S, Mazarati A, Shin D, et al. Inflammation enhances epileptogenesis in the developing rat brain. Neurobiol Dis. 2010;40(1):303-310.

18. Valera E, Masliah E. Immunotherapy for neurodegenerative diseases: Focus on $\alpha$-synucleinopathies. Pharmacology Therap. 2013;138(3):311-322.

19. Hutter-Saunders JA, Mosley RL, Gendelman HE. Pathways towards an effective immunotherapy for Parkinson's disease. Expert Rev Neurother. 2011;11(12):1703-1715.

20. Perry VH, Bell MD, Brown HC, et al. Inflammation in the nervous system. Curr Opin Neurobiol. 1995;5:636-641.

21. Aguzzi A, Barres BA, Bennett ML. Microglia: Scapegoat, saboteur, or something else? Science. 2013;339:156-161. 